Sharif University of Technology
Scientia Iranica
Transactions E: Industrial Engineering
h http://scientiairanica.sharif.edu
I RAN ICA

\title{
Developing mixed EWMA-CUSUM and CUSUM-EWMA control charts based on MRSS and DRSS procedures
}

\author{
A. Mohammadkhani and A. Amiri* \\ Department of Industrial Engineering, Shahed University, Tehran, Iran. \\ Received 14 March 2020; received in revised form 9 August 2020; accepted 3 October 2020
}
KEYWORDS
Average Run Length
(ARL);
Exponentially
Weighted Moving
Average (EWMA)
Cumulative Sum
(CUSUM);
Double Ranked Set
Sampling (DRSS);
Median Ranked Set
Sampling (MRSS);
Statistical Process
Monitoring (SPM).

\begin{abstract}
The mixed EWMA-CUSUM and CUSUM-EWMA control charts are among the control charts provided in recent years by combining two Exponentially Weighted Moving Average (EWMA) and Cumulative Sum (CUSUM) charts for efficient monitoring of the process mean. In this paper, these mixed control charts are extended using new median ranked set sampling and double ranked set sampling procedures. The performance of the proposed mixed control charts is evaluated through extensive Monte Carlo simulations in terms of average run length criterion. The results demonstrate that the proposed charts outperform similar ones in detecting different shifts in the process mean. Furthermore, a real dataset is also presented for explaining the implementation of the proposed control charts.
\end{abstract}

(C) 2022 Sharif University of Technology. All rights reserved.

\section{Introduction}

With the growing pressure from the market, companies need to remain competitive and cost-effective in production, which is the reason why being "qualitycentric" has become an essential area of focus at every level of a company. Quality is the main component that not only brings about greater financial gain for the company but also enhances the reputation of the organization in the market as a quality-centric organization. The quality of each product is affected by two types of variation in process parameters: common

*. Corresponding author. Tel.: +982151215240 E-mail addresses: atieh.mohamadkhani@yahoo.com (A. Mohammadkhani); amiri@shahed.ac.ir (A. Amiri)

doi: $10.24200 /$ sci.2020.55632.4328 causes of variation or special causes of variation. In any process, common causes of variations may be present, but assignable causes of variations are caused only by faults in the process, requiring immediate attention and corrective actions. Statistical Process Control (SPC) consists of tools that help monitor process parameters (location and dispersion) for both common and assignable causes of variation. Control chart is a helpful and powerful graphical technique of SPC for continuously monitoring the quality of a process. Each control chart is made of control limits, forming a confidence interval for the statistic monitored, the Upper Control Limit (UCL), the centerline (CL), and the Lower Control Limit (LCL). When the data come from an in-control process, the plotting points fall within the UCL and LCL. However, in an out-of-control process, one or more points fall outside the control limits. The chief purpose of each control chart is to identify the out- 
of-control situation. When the occurrence of special causes is recognized before producing a large number of defective products, necessary corrective actions for eliminating process variation and returning to the in-control situation can be taken immediately. The application of control charts to distinguish between common and assignable causes of variation in a process was initiated by Shewhart [1]. The Shewhart control chart is a memoryless control chart that does not consider the past relevant information; therefore, it is most commonly used to detect larger shifts in the process. For efficient detection of small and moderate shifts in the process, memory control charts such as Exponentially Weighted Moving Average (EWMA) and Cumulative Sum (CUSUM) control charts are the most suitable alternatives to Shewhart control chart. The EWMA control chart was introduced by Robert [2]; it weights observations in geometrically decreasing order so that the most recent observations contribute significantly, while the oldest observations contribute less. Page [3] designed the CUSUM control chart, which considered both past and current information and used the CUSUM of deviations from a target.

Increasing the use of memory control charts in various fields in recent times has sparked researchers' interest in modifying these control charts to enhance their efficiency for detecting different shifts in a process. Lucas [4] introduced the first combination of CUSUM and Shewhart control charts for detecting small and large shifts in the process. In the same way, Lucas and Saccucci [5] proposed the other mixed control chart and called it the Shewhart-EWMA control chart to enhance the EWMA control chart's performance. Jiang et al. [6] extended the ACUSUM chart by incorporating the EWMA-C estimators to detect the range of unknown shifts in the process location, in the proposed ACUSUM control chart, the reference values for both steady and zero states are updated with regarding EWMA-C control chart estimators. Abbas et al. [7] used the EWMA control chart in combination with the CUSUM control chart and named it MEC control chart. The EWMA control chart's statistics are used as the input of CUSUM control chart in the MEC control chart. Zaman et al. [8] designed MCE control chart as a reversed form of the MEC control chart. In their study, the CUSUM control chart's statistics are adopted as the input of EWMA control chart. Aslam [9] used the idea of Zaman et al. [8] and proposed MEC control chart for Weibull distribution. He suggested that the proposed chart can be utilized for the health departments to investigate the rate of infections. Osei-Aning et al. [10] used the modified control charts for monitoring the effects of autocorrelation in the process. They used the mixed MEC and MCE control charts for detecting all small, medium, and significant shifts in the process mean. Riaz [11] introduced MEC-TCC control chart with mixing the Tukey EWMA and Tukey CUSUM control charts. Based on their study, the MEC-TCC control chart performs well for skewed and symmetric distributions. Ajadi and Riaz [12] introduced multivariate control charts to enhance the performance of control charts for detecting the shifts in the process mean vector. Aslam et al. [13] extended the hybrid control charts, assuming that the quality characteristic follows Weibull distribution. They used the proposed mixed HEWMACUSUM control chart for detecting the process shifts in the healthcare department. Abbas et al. [14] adapted dual side CUSUM with EWM control charts to monitor the location process. They worked on a mixed EWMA Dual-CUSUM control chart to increase the sensitivity of control schemes used by analyzers in the petrochemical industry. Riaz et al. [15] proposed the MMECD by combining the structure of EWMA and CUSUM control charts. The MMECD control chart was introduced to obtain a sensitive scheme for detecting the shifts in the variance-covariance matrix of the process. Rao [16] constructed a mixed EWMA and CUSUM control charts to detect small shifts in the process location when the process follows the ConwayMaxwell (COM-Poisson) distribution.

Ranked Set Sampling (RSS) scheme was first developed by McIntyre [17], without considering theories and mathematical proof to estimate the mean of pasture and forage yields. The RSS scheme is highly beneficial and superior to the standard Simple Random Sampling (SRS) for estimation of the population mean. The RSS scheme has many applications in different fields including ecological and environmental studies, reliable theory, medical studies, and quality control. Salazar and Sinha [18] were the first ones who used this new sampling method for designing control charts; they used both RSS and Median Ranked Set Sampling (MRSS) methods for detecting shifts in the mean of a process. Later on, Muttlak and Al-Sabah [19] developed control charts based on RSS and MRSS data. Abujiya and Muttlak [20] and Mehmood et al. [21] used Double Ranked Set Sampling (DRSS) and MRSS methods, respectively. For improving the sensitivity of CUSUM control chart in detecting small shifts in the process mean, Al-Sabah [22] used RSS and MRSS methods. Abujiya and Lee [23] evaluated the performance of both memory and memory-less control charts using the RSS method. Haq et al. [24] improved the EWMA control chart by using the rank set sampling methods for monitoring the mean and variance of the process. Also, for some new researches, the readers can refer to Awasi and Haq [25], Haq and Munir [26], and Abbasi [27].

Following the previous research that aimed to improve the power of control charts in detecting small and medium shifts in the process mean, in this paper, we 
incorporate novel sampling methods including DRSS and MRSS in the combination of CUSUM and EWMA control charts. In other words, the main aim of this paper is to improve the performance of the previous control charts in detecting small and medium shifts. To validate the performance of the proposed control charts, this study uses Monte Carlo simulation runs and exhibits the superiority of the proposed charts in terms of Average Run Length (ARL) criterion.

To this end, a quality characteristic with normal distribution is considered here. Also, it is assumed that the parameters of the process are known; we are at Phase-II monitoring of the process and the process variance is constant over time. Then, two new control charts are proposed. The structure of this paper hereafter is as follows: In Section 2, the notations and definitions are introduced. The sampling methods including SRS, RSS, DRSS, and MRSS are briefly introduced in Section 3. Section 4 describes the classic MEC and MCE control charts. Section 5 presents the proposed control charts. A comprehensive comparative evaluation of the proposed control charts and similar classical control charts based on the SRS and RSS methods is presented in Section 6 . Section 7 considers a case to demonstrate the applicability of the proposed MEC and MCE charts. The conclusion and suggestions are given in Section 8 .

\section{Notations and assumptions}

In this section, to facilitate the presented models, some notations and assumptions are presented.

SRS: Simple Random Sampling;

RSS: Ranked Set Sampling;

DRSS : Double Ranked Set Sampling;

MRSS: Median Ranked Set Sampling;

MEC: Mixed EWMA-CUSUM;

MCE: Mixed CUSUM-EWMA;

$A R L_{0}$ : The in-control Average Run length;

$A R L_{1}$ : The out-of-control Average Run length;

$m$ : Sample size;

$X_{i}: i$ th observation in a random sample;

$X_{j i}: i$ th unit of $j$ th sets;

$X_{j(i: m)}: i$ th ordered statistic from the $j$ th set by the size $m$;

$X_{j(i: m)}^{(k)}: i$ th ordered statistic in the $j$ th set by the size $m$ of the $k$ th RSS sample;

$f_{(i: m)}(x)$ : The probability distribution function of the $i$ ordered statistic;

$F_{(i: m)}(x)$ : The cumulative distribution function of the $i$ ordered statistic;

$\mu$ : The population mean;

$\mu_{0}$ : The mean of the process when the process is incontrol; $\sigma$ : The population standard deviation;

$\bar{X}_{S R S}$ : The SRS estimator of the population mean;

$\bar{X}_{R S S}$ : The RSS estimator of the population mean;

$\bar{X}_{D R S S}$ : The DRSS estimator of the population mean;

$\bar{X}_{M R S S}$ : The MRSS estimator of the population mean; $a^{\prime}$ : The reference value in the CUSUM control chart and the MEC control chart;

$m^{\prime}$ : The reference value in the CUSUM control chart and the MEC-MRSS control chart;

$d^{\prime}$ : The reference value in the CUSUM control chart and the MEC-DRSS control chart;

$k$ : The reference value in the classic CUSUM control chart;

$Z_{t}$ : The EWMA statistic;

$a_{t}$ : The time varying reference value in the MEC control chart;

$d_{t}$ : The time varying reference value in the MEC-DRSS control chart;

$m_{t}$ : The time varying reference value in the MECMRSS control chart;

$L_{C}$ : The coefficient of the MCE chart control limit;

$\lambda$ : The smoothing parameter of MEC and MCE control charts;

$\lambda_{m}$ : The smoothing parameter of MEC-DRSS and MEC-MRSS control charts;

$\lambda_{c}$ : The smoothing parameter of MCE-DRSS and MCE-MRSS control charts;

$\delta$ : The magnitude shift in mean;

$U C L_{(M E C)_{t}}$ : The control limit of the MEC control chart;

$U C L_{(M C E)_{t}}$ : The control limit of the MCE control chart;

$U C L_{(M E C-D R S S)_{t}}$ : The control limit of the MECDRSS control chart;

$U C L_{(M C E-D R S S)_{t}}$ : The control limit of the MCEDRSS control chart;

$U C L_{(M E C-M R S S)_{t}}$ : The control limit of the MECMRSS control chart;

$U C L_{(M C E-M R S S)_{t}}$ : The control limit of the MCEMRSS control chart.

Also, in this paper, it is assumed that:

- The parameters of the process are known;

- The monitoring of the process is at Phase-II;

- The process variance is constant over time; and

- The quality characteristic follows normal distribution.

\section{Structure of sampling methods}

In this section, the sampling procedures including SRS, RSS, DRSS, and MRSS are explained and detailed as follows. 


\subsection{Simple Random Sampling (SRS)}

A sample is defined as an SRS of size $m$ if selected, such that the observations $\left\{X_{i}\right\}$ are independently and identically distributed. Let $X_{1}, X_{2}, \ldots, X_{m}$ represent the observations in a random sample drawn from a distribution having the probability distribution function $\mathrm{f}(\mathrm{x})$ and cumulative distribution function $\mathrm{F}(\mathrm{X})$ with mean $\mu$ and variance $\sigma$. Then, the SRS estimator of the population mean $\left(\bar{X}_{S R S}\right)$ and the variance of $\bar{X}_{S R S}$ are defined as follows:

$$
\begin{gathered}
\bar{X}_{S R S}=\frac{1}{m} \sum_{i=1}^{m} X_{i}, \\
\operatorname{Var}\left(\bar{X}_{S R S}\right)=\frac{\sigma^{2}}{m} .
\end{gathered}
$$

\subsection{Ranked Set Sampling (RSS)}

An RSS method is a random selection of samples from a large community or population such that everyone in the sample has an equal chance to be chosen [28]. RSS is a cost-effective sampling method compared to SRS scheme. In the case of SRS, the sample size must increase to enhance the chance of coverage of the entire observation values. In the RSS method, representation can be increased based on a certain number of sample observations. Hence, significant saving in the measurement cost is expected.

The RSS sampling procedure is defined based on the following steps:

Step 1: Select $m^{2}$ units of the process randomly;

Step 2: Assign these $m^{2}$ units to $m$ sets, each set of size $m$;

Step 3: Rank units of each set regarding the process variable;

Step 4: Select the smallest ranked unit of the first set, the second ranked unit of the second set, and the $i$ th ranked unit of the $i$ th set. The process continues in this way up until the $m$ th smallest ranked unit of the last set. This is a full cycle of the RSS by sample size $m[17]$.

Let $X_{11}, \ldots, X_{1 m} ; X_{21}, \ldots, X_{2 m} ; X_{m 1}, \ldots, X_{m m}$ denote independent random samples of size $m$ with the same $f(x)$ and $F(x)$. The set of $X_{1(1: m)}$, $X_{2(2: m)}, \ldots, X_{m(m: m)}$ represents the final set which is considered as an evaluation sample; $X_{i(i: m)}$ defines the ordered statistic, which indicates the $i$ th unit of the $i$ th set by size $m$ regarding the RSS. This means that $X_{1(1: m)}$ is the smallest unit from the first set; $X_{2(2: m)}$ is the second smallest unit from another set; and $X_{m(m: m)}$ is the largest ranked unit from the last set.

To clarify the sampling method, an example is given here. Let $m=3$; thus, we need to identify $m^{2}=9$ units randomly from the population. Then, these $m^{2}$ units are allocated to $m$ sets with size $m$ and each set will be ranked with respect to the variables of interest; $X_{j(i: m)}$ is the $i$ th $(i=1,2,3)$ smallest units in the $j$ th $(j=1,2,3)$ set. Then, the $i$ th $(i=1,2,3)$ ranked unit of the $i$ th set will be selected; this final step yields the RSS set with size $m$. The following process indicates the steps of the RSS method:

\section{Step 1:}

$$
\left[X_{1}, X_{2}, X_{3}, X_{4}, X_{5}, X_{6}, X_{7}, X_{8}, X_{9}\right]
$$

\section{Step 2:}

$$
\begin{aligned}
& \text { Set } \\
& 1 \\
& 2 \\
& 3
\end{aligned}\left[\begin{array}{lll}
X_{11} & X_{12} & X_{13} \\
X_{21} & X_{22} & X_{23} \\
X_{31} & X_{32} & X_{33}
\end{array}\right],
$$

Step 3:

$$
\begin{aligned}
& \text { Set } \\
& 1 \\
& 2 \\
& 3
\end{aligned}\left[\begin{array}{lll}
X_{1(1: 3)} & X_{1(2: 3)} & X_{1(3: 3)} \\
X_{2(1: 3)} & X_{2(2: 3)} & X_{2(3: 3)} \\
X_{3(1: 3)} & X_{3(2: 3)} & X_{3(3: 3)}
\end{array}\right],
$$

\section{Step 4:}

$$
\begin{aligned}
& \text { Set } \\
& \left.\begin{array}{llll}
1 & \multicolumn{1}{l}{} & \\
2 & X_{1(1: 3)} & X_{1(2: 3)} & X_{1(3: 3)} \\
3 & X_{2(1: 3)} & X_{2(2: 3)} & X_{2(3: 3)} \\
X_{3(1: 3)} & X_{3(2: 3)} & X_{3(3: 3)}
\end{array}\right] .
\end{aligned}
$$

Thus, the $\left\{X_{1(1: 3)}, X_{2(2: 3)}, X_{3(3: 3)}\right\}$ is an RSS sample of size $m=3$.

For $X_{i(i: m)}$, the probability distribution function $f_{(i: m)}(x)$ and cumulative distribution function $F_{(i: m)}(x)$ are given orderly as follows:

$f_{(i: m)}(x)=m\left(\begin{array}{l}m-1 \\ i-1\end{array}\right)\{F(x)\}^{i-1}\{1-F(x)\}^{m-i} f(x)$,

and:

$F_{(i: m)}(x)=\sum_{l=i}^{m}\{F(x)\}^{l}\{1-F(x)\}^{m-l}, \quad-\infty<x<\infty$.

Let $\bar{X}_{R S S}=\frac{1}{m} \sum_{i=1}^{m} X_{i(i: m)}$ be the RSS sample mean and $\mu$ and $\sigma$ are the population mean and population standard deviation, respectively. The mean and variance of the population based on RSS method are given by [29]:

$$
\begin{aligned}
\mathrm{E}\left[\bar{X}_{R S S}\right]= & \frac{1}{m} \sum_{i=1}^{m} \mathrm{E}\left[X_{i(i: m)}\right], \\
\operatorname{Var}\left(\bar{X}_{R S S}\right) & =\frac{1}{m^{2}}\left\{m \sigma^{2}-\sum_{i=1}^{m}\left(\mu_{i(i: m)}-\mu\right)^{2}\right\} \\
& =\frac{\sigma^{2}}{m}-\frac{1}{m^{2}} \sum_{i=1}^{m}\left(\mu_{(i: m)}-\mu\right)^{2} .
\end{aligned}
$$




\subsection{Double Ranked Set Sampling (DRSS)}

As an extension of RSS method, the DRSS scheme was presented by Al-Saleh and Al-Kadiri [30]. In the RSS scheme, the efficiency of $\bar{X}_{R S S}$ is related to the size of $m$ upon increasing the sample size, a more efficient sample will be obtained; in practice, for the large value of $m$ accurate visual ranking will be difficult. Therefore, for an instant $m$ value, ranking in the second stage is more efficient than the first stage. The DRSS procedure with size $m$ can be described as follows:

Step 1: Randomly select $m^{3}$ units from the target population and divide them into $m$ sets with the size $m^{2}$;

Step 2: Perform usual RSS method on each $\mathrm{m}^{2}$ set to obtain $m$ RSS sets;

Step 3: Apply the RSS method again to $m$ RSS set by size $m$ to obtain a DRSS set with the sample size $m$.

$$
\text { Let }\left\{X_{11}^{(1)}, X_{12}^{(1)}, \ldots, X_{m m}^{(1)}\right\},\left\{X_{11}^{(2)}, X_{12}^{(2)}, \ldots, X_{m m}^{(2)}\right\},
$$
$\ldots,\left\{X_{11}^{(m)}, X_{12}^{(m)}, \ldots, X_{m m}^{(m)}\right\}$ be $m^{2}$ independent random samples with the size $m . X_{j i}^{(k)}$ is the $j$ th observation of the $k$ th RSS sample in the $j$ th set. After ranking these units, $m$ RSS sets are obtained and for each value of $m$, we have $\left\{X_{1(1: m)}^{(k)}, X_{2(2: m)}^{(k)}, \ldots, X_{m(m: m)}^{(k)}\right\}$ for $k=$ $1,2, \ldots, m . X_{i(i: m)}^{(k)}$ is the $i$ th ordered observation in the set of the $k$ th RSS sample.

To clarify the DRSS method, an example with a sample with size $m=3$ is given here. In this case, we must randomly select $m^{3}=27$ units from the population and divide them into 3 sets with size 9 . After visually ranking the elements of three sets, three RSS sets are obtained. Then, for each obtained RSS set, the RSS procedure is obtained again:

\section{Step 1:}

$$
\begin{array}{r}
{\left[X_{11}^{(1)}, X_{12}^{(1)}, \cdots, X_{33}^{(1)}, X_{11}^{(2)}, X_{12}^{(2)}, \cdots,\right.} \\
\left.X_{33}^{(2)}, X_{11}^{(3)}, X_{12}^{(3)}, \cdots, X_{33}^{(3)}\right],
\end{array}
$$

\section{Step 2:}

$$
\begin{aligned}
& \text { Set }
\end{aligned}
$$

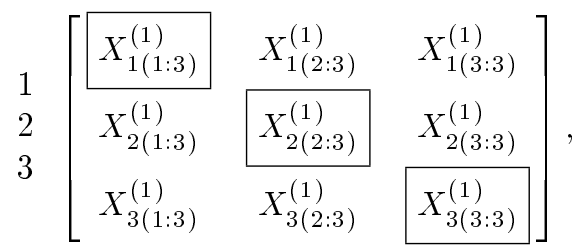

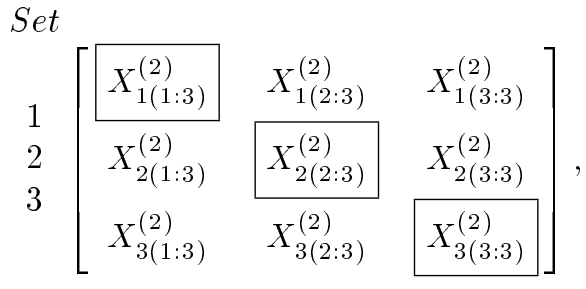

$$
\begin{aligned}
& \text { Set } \\
& 1 \\
& 2 \\
& 3 \\
& \left.\begin{array}{ccc}
X_{1(1: 3)}^{(3)} & X_{1(2: 3)}^{(3)} & X_{1(3: 3)}^{(3)} \\
X_{2(1: 3)}^{(3)} & X_{2(2: 3)}^{(3)} & X_{2(3: 3)}^{(3)} \\
X_{3(1: 3)}^{(3)} & X_{3(2: 3)}^{(3)} & X_{3(3: 3)}^{(3)}
\end{array}\right],
\end{aligned}
$$

Step 3:

$$
\begin{aligned}
& R_{1}=\left\{X_{1(1: 3)}^{(1)}, X_{2(2: 3)}^{(1)}, X_{3(3: 3)}^{(1)}\right\}, \\
& R_{2}=\left\{X_{1(1: 3)}^{(2)}, X_{2(2: 3)}^{(2)}, X_{3(3: 3)}^{(2)}\right\}, \\
& R_{3}=\left\{X_{1(1: 3)}^{(3)}, X_{2(2: 3)}^{(3)}, X_{3(3: 3)}^{(3)}\right\} .
\end{aligned}
$$

So $\left\{X_{1(1: 3)}^{(1)}, X_{2(2: 3)}^{(2)}, X_{3(3: 3)}^{(2)}\right\}$ is a DRSS sample with size $m=3$.

For a DRSS sample of size $m$, the sample mean is given by Al-Saleh and Al-Kadiri [28]:

$$
\bar{X}_{D R S S}=\frac{1}{m} \sum_{i=1}^{m} \mu_{(i: m)},
$$

with variance:

$$
\operatorname{Var}\left(\bar{X}_{D R S S}\right)=\frac{1}{m} \sum_{i=1}^{m} \sigma_{(i: m)}^{2}+\frac{1}{m} \sum_{i=1}^{m}\left(\mu_{(i: m)}-\mu\right)^{2} .
$$

\subsection{Median Ranked Set Sampling (MRSS)}

MRSS is an appropriate method for symmetric distribution around $\mu$. This method was presented by Muttlak [31]. He introduced this method to reduce the error rate in the estimation of the population mean. The structure of the MRSS method is as follows:

Step 1: Select $m^{2}$ units of the process randomly;

Step 2: Partition $m^{2}$ units of the target population into $m$ sets with size $m$;

Step 3: Rank units of each set through visual inspection regarding the process variable;

Step 4: After ranking the divided units, there would be two possibilities. If $m$ is even, the $m / 2$ th smallest ranked units of the $m / 2$ th first units and the $(m+2) / 2$ th smallest ranked units of the $m / 2$ th last units are selected. If $m$ is odd, the $(m+1) / 2$ th smallest ranked units of all units are selected. This is an MRSS sample with size $m$. 
For clarification of the MRSS scheme, an example is given. We assume that an odd sample, of size $m^{2}=9$, is selected from the observed population. This selected sample is divided independently into 3 sets with size 3 and then, the divided units are ranked. From Step 4 of MRSS ranking method, when the sample size is odd, the second smallest ranked units of all units should be selected.

\section{Step 1:}

$$
\left[X_{1}, X_{2}, X_{3}, X_{4}, X_{5}, X_{6}, X_{7}, X_{8}, X_{9}\right],
$$

\section{Step 2:}

$$
\begin{aligned}
& \text { Set } \\
& 2 \\
& 3 \\
& 3
\end{aligned}\left[\begin{array}{lll}
X_{11} & X_{12} & X_{13} \\
X_{21} & X_{22} & X_{23} \\
X_{31} & X_{32} & X_{33}
\end{array}\right]
$$

\section{Step 3:}

$$
\begin{aligned}
& \text { Set } \\
& 1 \\
& 2 \\
& 3
\end{aligned}\left[\begin{array}{lll}
X_{1(1: 3)} & X_{1(2: 3)} & X_{1(3: 3)} \\
X_{2(1: 3)} & X_{2(2: 3)} & X_{2(3: 3)} \\
X_{3(1: 3)} & X_{3(2: 3)} & X_{3(3: 3)}
\end{array}\right],
$$

\section{Step 4:}

$$
\begin{aligned}
& \text { Set } \\
& \left.\begin{array}{ll|l|l}
1 & X_{1(1: 3)} & X_{1(2: 3)} & X_{1(3: 3)} \\
3 & X_{2(1: 3)} & X_{2(2: 3)} & X_{2(3: 3)} \\
X_{3(1: 3)} & X_{3(2: 3)} & X_{3(3: 3)}
\end{array}\right] .
\end{aligned}
$$

Hence, $\left\{X_{1(2: 3)}, X_{2(2: 3)}, X_{3(2: 3)}\right\}$ defines an MRSS sample with size $m=3$.

Let $X_{i((m+1) / 2: m)}$ denote the $(m+1) / 2$ th of the $i$ th sample for the odd set and for the even set, $X_{i(m / 2: m)}$ and $X_{i(m / 2+1: m)}$ denote the $m / 2$ th and $m / 2+1$ th order statistics of the $i$ th sample. Then, the population mean estimators of the MRSS for both even and odd sample sizes $m$ are given as follows:

$$
\begin{aligned}
& \bar{X}_{M R S S, E}=\frac{1}{m} \sum_{i=1}^{m / 2} X_{i(m / 2: m)}+\sum_{i=m / 2+1}^{m} X_{i(m / 2+1: m)} \\
& \bar{X}_{M R S S, O}=\frac{1}{m} \sum_{i=1}^{m} X_{i((m+1) / 2: m)}
\end{aligned}
$$

with variance:

$$
\begin{aligned}
& \operatorname{Var}\left(\bar{X}_{M R S S, E}\right)=\frac{1}{2 m}\left(\sigma_{x(m / 2: m)}^{2}+\sigma_{x(m / 2+1: m)}^{2}\right) \text { and } \\
& \operatorname{Var}\left(\bar{X}_{M R S S, O}\right)=\frac{1}{m} \sigma_{x((m+1) / 2: m)}^{2} .
\end{aligned}
$$

\section{MEC and MCE control charts}

In this section, the classical mixed CUSUM and EWMA control charts are briefly described.

\subsection{MEC control chart}

MEC is designed by integrating the EWMA and CUSUM control charts statistics to improve the performance of these control charts. Two integrated statistics of the CUSUM control charts are defined as follows:

$$
\begin{aligned}
& M E C_{t}^{+}=\max \left[0,\left(Z_{t}-\mu_{0}\right)-a_{t}+M E C_{t-1}^{+}\right], \\
& M E C_{t}^{-}=\max \left[0,-\left(Z_{t}-\mu_{0}\right)-a_{t}+M E C_{t-1}^{-}\right] .
\end{aligned}
$$

The initial values of the statistics in Eq. (11) are assumed to be equal to zero $M E C_{0}^{+}=M E C_{0}^{-}=0$. $a_{t}$ is the time varying reference value and is defined as follows:

$$
\begin{aligned}
a_{t} & =a^{\prime} \times \sqrt{\operatorname{Var}\left(Z_{t}\right)} \\
& =a^{\prime} \sigma_{\bar{X}} \sqrt{\frac{\lambda}{2-\lambda}\left(1-(1-\lambda)^{2 t}\right)},
\end{aligned}
$$

where $a^{\prime}$ is defined as the reference value of the CUSUM control chart. $M E C_{0}^{+}$and $M E C_{0}^{-}$are the upper-sided and the lower-sided CUSUM statistics, respectively. $M E C_{0}^{+}=M E C_{0}^{-}=0$ shares the initial value of CUSUM and $Z_{t}$ is the EWMA statistic and is defined by:

$$
Z_{t}=(1-\lambda) Z_{t-1}+\lambda \bar{X}_{t}
$$

where $\lambda$ is a constant value such that $0<\lambda \leq 1$. The starting value of $Z_{0}=\mu_{0}$ is given. The mean and variance of the statistic $Z_{t}$ are given as:

$$
\begin{aligned}
& E\left(Z_{t}\right)=\mu_{0} \\
& \operatorname{Var}\left(Z_{t}\right)=\frac{\sigma_{0}^{2}}{m}\left(\frac{\lambda}{2-\lambda}\left(1-(1-\lambda)^{2 t}\right)\right) .
\end{aligned}
$$

$U C L_{(M E C) t}$ is the control limit of the MEC control chart. If $M E C_{t}^{+}$or $M E C_{t}^{-}$falls outside $U C L_{(M E C) t}$, the process is out-of-control; otherwise, it is in-control. $U C L_{(M E C) t}$ is given as:

$$
\begin{aligned}
U C L_{(M E C) t} & =b^{\prime} \times \sqrt{\operatorname{Var}\left(Z_{t}\right)} \\
= & b^{\prime} \sigma_{\bar{X}} \sqrt{\frac{\lambda}{2-\lambda}\left(1-(1-\lambda)^{2 t}\right)} .
\end{aligned}
$$

The values $a_{t}$ and $b_{t}$ are constant values that are determined based on the standard deviation of the EWMA statistic. At a fixed value of $a^{\prime}$ the value of $b^{\prime}$ can be determined through simulation to obtain the desired $A R L_{0}$. Mostly, $a^{\prime}$ is considered to be half of the shift of standard deviation $Z_{t}$. 


\subsection{MCE control chart}

The MCE control chart is another mixture of the features of two CUSUM and EWMA control charts. The two plotting statistics of the MCE control chart are defined based on the CUSUM statistics.

$$
\begin{aligned}
& M C E_{t}^{+}=(1-\lambda) M C E_{t-1}^{+}+\lambda C_{t}^{+}, \\
& M C E_{t}^{-}=(1-\lambda) M C E_{t-1}^{-}+\lambda C_{t}^{-},
\end{aligned}
$$

where $C_{t}^{+}$and $C_{t}^{-}$are the classic CUSUM statistics and $0<\lambda \leq 1$ is the smoothing parameter. The initial values for the statistics $M C E_{t}^{+}$and $M C E_{t}^{-}$are equal to the target mean of $C_{t}^{+}$and $C_{t}^{-}\left(M C E_{0}^{+}=\right.$ $\left.M C E_{0}^{-}=\mu_{C}\right)$. For an in-control process, the values of mean and the variance of the CUSUM statistics may change throughout a set period of $t$, so that when $t$ approaches infinity, they are equal to constant values.

$$
\begin{aligned}
& E\left(C_{t}^{+}\right)=E\left(C_{t}^{-}\right)=\mu_{C}, \\
& \operatorname{Var}\left(C_{t}^{+}\right)=\operatorname{Var}\left(C_{t}^{-}\right)=\sigma_{C}^{2} .
\end{aligned}
$$

The control limit of $M E C^{-}$and $M E C^{+}$is calculated based on the expected value and variance of CUSUM statistics and is given as follows:

$$
U C L_{(M C E) t}=\mu_{C}+L_{C} \sigma_{C} \sqrt{\frac{\lambda}{2-\lambda}\left(1-(1-\lambda)^{2 t}\right)},
$$

where $L_{C}$ is the coefficient of the control limit.

\section{Proposed mixed control charts}

This section proposes new modified EWMA and CUSUM control charts, namely MEC-DRSS, MCEDRSS, MEC-MRSS, and MCE-MRSS. These control charts are based on combining the mixed MEC and MCE control charts with DRSS and MRSS methods.

\subsection{MEC control chart with DRSS}

The MEC-DRSS control chart is designed by integrating the design structure features of EWMA-DRSS and CUSUM control charts. $M E C_{D R S S, t}^{+}$and $M E C_{D R S S, t}^{-}$ are defined as the statistics of the proposed MEC-DRSS control chart and initially set to zero $\left(M E C_{D R S, 0}^{-}=\right.$ $\left.M E C_{D R S S, 0}^{+}=0\right)$ :

$$
\begin{aligned}
& M E C_{D R S S, t}^{+}= \\
& \max \left[0,\left(Z_{D R S S, t}-\mu_{0}\right)-d_{t}+M E C_{D R S S, t-1}^{+}\right], \\
& M E C_{D R S S, t}^{-}= \\
& \quad \max \left[0,-\left(Z_{D R S S, t}-\mu_{0}\right)-d_{t}+M E C_{D R S S, t-1}^{-}\right]
\end{aligned}
$$

Like MEC, the time-varying reference value of the MEC-DRSS is given as:

$$
d_{t}=d^{\prime} \times \sqrt{\operatorname{Var}\left(Z_{D R S S, t}\right)} .
$$

Based on the DRSS method, the EWMA statistic $Z_{D R S S, t}$ is defined below:

$$
Z_{D R S S, t}=\lambda_{m} \bar{X}_{D R S S, t}+\left(1-\lambda_{m}\right) Z_{D R S S, t-1},
$$

where $\bar{X}_{D R S S}$ is the mean of the sample based on DRSS method and can be calculated using the method explained in Section 3.3, and $\lambda_{m}$ is a constant value $\left(0<\lambda_{m} \leq 1\right)$ like $\lambda$ in Section 4 . The starting value of $Z_{D R S S}$ is set to zero with the mean and variance:

$$
\begin{aligned}
E\left(Z_{D R S S, t}\right)=\mu_{0} & \\
\operatorname{Var}\left(Z_{D R S S, t}\right)= & {\left[\frac{\lambda_{m}}{2-\lambda_{m}}\left(1-\left(1-\lambda_{m}\right)^{2 t}\right)\right] } \\
& \operatorname{Var}\left(\bar{X}_{D R S S, t}\right) .
\end{aligned}
$$

The statistics $M E C_{D R S S, 0}^{+}$and $M E C_{D R S S, 0}^{-}$are plotted against the control limit $U C L_{(M E C-D R S S) t}$.

$$
U C L_{(M E C-D R S S) t}=D^{\prime} \times \sqrt{\operatorname{Var}\left(Z_{D R S S, t}\right)} .
$$

For a fixed value of $d^{\prime}$, the value of $D^{\prime}$ can be determined using simulation to obtain the desired $A R L_{0}$.

\subsection{MCE control chart with DRSS}

The MCE-DRSS control chart is the reverse format of the proposed MCE-DRSS control chart. This chart has been designed based on the statistics of CUSUMDRSS, $C_{D R S S, t}^{+}$and $C_{D R S S, t}^{-}$. Therefore, the two statistics of MCE-DRSS are defined as follows:

$$
\begin{aligned}
& M C E_{D R S S, t}^{+}=\lambda_{c} C_{D R S S, t}^{+}+\left(1-\lambda_{c}\right) M C E_{D R S S, t-1}^{+}, \\
& M C E_{D R S S, t}^{-}=\lambda_{c} C_{D R S S, t}^{-}+\left(1-\lambda_{c}\right) M C E_{D R S S, t-1}^{-},
\end{aligned}
$$

where $0<\lambda_{C} \leq 1$ is the smoothing parameter.

$$
\begin{aligned}
& C_{D R S S, t}^{+}= \\
& \max \left[0,\left(\bar{X}_{D R S S, t}-\mu_{0}\right)-k+C_{D R S S, t-1}^{+}\right] \\
& C_{D R S S, t}^{-}= \\
& \max \left[0,-\left(\bar{X}_{D R S S, t}-\mu_{0}\right)-k+C_{D R S S, t-1}^{-}\right],
\end{aligned}
$$

where $k$ is the reference value and generally taken as half of the magnitude of the difference between the values $\mu_{0}$ and $\mu_{1}$. If the shift $\delta$ in units of standard 
deviation is defined as $\delta=\left|\mu_{1}-\mu_{0}\right| / \sigma_{\bar{X}_{D R S S}}$, then $k$ is defined as follows:

$$
k=\frac{\delta}{2} \sigma_{\bar{X}_{D R S S}}=\frac{\left|\mu_{1}-\mu_{0}\right|}{2} .
$$

The initial values of $M C E_{D R S S, t}^{+}$and $M C E_{D R S S, t}^{-}$are set to the in-control mean CUSUM-DRSS statistics $\left(M C E_{D R S S, 0}^{+}=M C E_{D R S S, 0}^{-}=\mu_{c_{D R S S}}\right)$. If $t$ approaches infinity, the mean and the variance of the CUSUM-DRSS statistics will be constant.

$$
\begin{aligned}
& E\left(C_{D R S S, t}^{+}\right)=E\left(C_{D R S S, t}^{-}\right)=\mu_{c_{D R S S}}, \\
& \operatorname{Var}\left(C_{D R S S, t}^{+}\right)=\operatorname{Var}\left(C_{D R S S, t}^{-}\right)=\sigma_{c_{D R S S}}^{2} .
\end{aligned}
$$

Hence, the control limit of the MCE-DRSS is defined as follows:

$$
\begin{aligned}
& U C L_{(M C E-D R S S) t}=\mu_{c_{D R S S}} \\
& \quad+L_{C} \sigma_{c_{D R S} S} \sqrt{\frac{\lambda}{2-\lambda}\left(1-\left(1-\lambda_{c}\right)^{2 t}\right)}
\end{aligned}
$$

where $L_{C}$ is the coefficient of control limit and determined such that the overall predefined $A R L_{0}$ is obtained. As soon as $M C E_{D R S S, t}^{+}>U C L_{(M C E-D R S S) t}$ or $M C E_{D R S S, t}^{-}>U C L_{(M C E-D R S S) t}$, an alarm is triggered and the process will be out-of-control. If $M C E_{D R S S, t}^{+}>U C L_{(M C E-D R S S) t}$, an upward mean shift occurs. Similarly, if $M C E_{D R S S, t}^{-}>$ $U C L_{(M C E-D R S S) t}$, a downward mean shift occurs.

\subsection{MEC control chart with MRSS}

In this chart, the CUSUM control chart is defined by the statistics of the EWMA based on MRSS method. The two plotting statistics of MEC-MRSS are as follows:

$$
\begin{aligned}
& M E C_{M R S S, t}^{+}= \\
& \max \left[0,\left(Z_{M R S S, t}-\mu_{0}\right)-m_{t}+M E C_{M R S S, t-1}^{+}\right], \\
& M E C_{M R S S, t}^{-}= \\
& \quad \max \left[0,-\left(Z_{M R S S, t}-\mu_{0}\right)-m_{t}+M E C_{M R S S, t-1}^{-}\right],
\end{aligned}
$$

where $M E C_{M D R S S, 0}^{-}=M E C_{M D R S S, 0}^{+}=0$. Also, $m_{t}$ is the time-varying reference value of MEC-MRSS:

$$
m_{t}=m^{\prime} \times \sqrt{\operatorname{Var}\left(Z_{M R S S, t}\right)},
$$

where $m^{\prime}$ is the reference value in the CUSUM control chart.

Based on the statistic of EWMA and MRSS method, $Z_{M R S S, t}$ is given as:

$$
Z_{M R S S, t}=\lambda_{m} \bar{X}_{M R S S, t}+\left(1-\lambda_{m}\right) Z_{M R S S, t-1} .
$$

$\bar{X}_{M R S S}$ is the mean of a median ranked set, which is calculated based on the method elaborated in Section 3.4. $Z_{M R S S, 0}=0$ is the initial value. The mean and variance of the statistic $Z_{M R S S}$ are given as follows:

$$
\begin{aligned}
E\left(Z_{M R S S, t}\right)= & \mu_{0}, \\
\operatorname{Var}\left(Z_{M R S S, t}\right)= & {\left[\frac{\lambda_{m}}{2-\lambda_{m}}\left(1-\left(1-\lambda_{m}\right)^{2 t}\right)\right] } \\
& \operatorname{Var}\left(\bar{X}_{M R S S, t}\right) .
\end{aligned}
$$

The statistics $M E C_{M R S S, 0}^{+}$and $M E C_{M R S S, 0}^{-}$are plotted against the control limit $U C L_{(M E C-M R S S) t}$.

$$
U C L_{(M E C-M R S S) t}=M^{\prime} \times \sqrt{\operatorname{Var}\left(Z_{M R S S, t}\right)} .
$$

\subsection{MCE control chart with MRSS}

MCE-MRSS is designed based on the CUSUM-MRSS as an input for the EWMA control chart and the statistics are as follows:

$$
\begin{aligned}
& M C E_{M R S S, t}^{+}=\lambda_{c} C_{M R S S, t}^{+} \\
& \quad+\left(1-\lambda_{c}\right) M C E_{M R S S, t-1}^{+}, \\
& M C E_{M R S S, t}^{-}=\lambda_{c} C_{M R S S, t}^{-} \\
& \quad+\left(1-\lambda_{c}\right) M C E_{M R S S, t-1}^{-},
\end{aligned}
$$

where $0<\lambda_{C} \leq 1$ is the smoothing parameter, and $C_{M R S S, t}^{+}$and $C_{M R S, t}^{-}$are the CUSUM-MRSS statistics and defined as follows:

$$
\begin{aligned}
& C_{M R S S, t}^{+}= \\
& \max \left[0,\left(\bar{X}_{M R S S, t}-\mu_{0}\right)-k+C_{M R S S, t-1}^{+}\right] \\
& C_{M R S S, t}^{-}= \\
& \max \left[0,-\left(\bar{X}_{M R S S, t}-\mu_{0}\right)-k+C_{M R S S, t-1}^{-}\right]
\end{aligned}
$$

where $k$ is called the reference value and is usually chosen as about half of the difference between incontrol value $\mu_{0}$ and the out-of-control value $\mu_{1}$. If the shift $\delta$ in the unit of standard deviation is defined as $\delta=\left|\mu_{1}-\mu_{0}\right| / \sigma_{\bar{X}_{M R S}}$, then $k$ is half of the shift or:

$$
k=\frac{\delta}{2} \sigma_{\bar{X}_{M R S S}}=\frac{\left|\mu_{1}-\mu_{0}\right|}{2} .
$$

The starting values of $M C E_{M R S S, t}^{+}$and $M C E_{M R S S, t}^{-}$ are set to the in-control mean CUSUM-MRSS statistics $\left(M C E_{M R S S, 0}^{+}=M C E_{M R S S, 0}^{-}=\mu_{c_{M R S S}}\right)$. If $t$ 
approaches infinity, the mean and variance of $C_{M R S S, t}^{+}$ and $C_{M R S, t}^{-}$will become constant.

$$
\begin{aligned}
& E\left(C_{M R S S, t}^{+}\right)=E\left(C_{M R S S, t}^{-}\right)=\mu_{c_{M R S S}}, \\
& \operatorname{Var}\left(C_{M R S S, t}^{+}\right)=\operatorname{Var}\left(C_{M R S S, t}^{-}\right)=\sigma_{c_{M R S S}}^{2},
\end{aligned}
$$

where $\mu_{c_{M R S S}}$ and $\sigma_{c_{M R S S}}$ are the mean and variance of CUSUM-MRSS. The control limit of the MCE-MRSS is defined as follows:

$$
\begin{aligned}
U C L_{(M C E-M R S S) t} & =\mu_{c_{M R S S}} \\
& +L_{C} \sigma_{c_{M R S S}} \sqrt{\frac{\lambda}{2-\lambda}\left(1-\left(1-\lambda_{c}\right)^{2 t}\right)},
\end{aligned}
$$

where $L_{C}$ is the coefficient of MCE-MRSS, which is defined using simulation to obtain the desired $A R L_{0}$.

\section{Performance comparison}

This section provides a detailed comparison of the proposed mixed MEC and MCE control charts with some existing schemes in terms of ARL to investigate the performance of the proposed control charts. ARL is one of the measures for judging the performance of control charts. At Phase II, two types of ARL are used, that is, in-control $A R L\left(A R L_{0}\right)$ and out-of-control $A R L\left(A R L_{1}\right)$. A control chart is efficient as long as $A R L_{1}$ is small enough to detect a shift as quickly as possible at a fixed value of $A R L_{0}$. In the literature, different approaches including Monte Carlo method, Markov Chain Approach (MCA), Martingale approach, explicit formulas, and numerical integral equation for evaluating ARL are described. In this paper, the Monte Carlo simulation approach is used for the evaluation procedure. The simulation iterates 50,000 times for calculating the ARL values and the algorithm is applied using MATLAB. Tables 1-4 are constructed for out-ofcontrol run length characteristics of the proposed MEC and MCE control charts for controlling the sample mean by assuming that the quality characteristics follow normal distribution. $A R L_{0}$ is considered equal to 200 and the reference value for the CUSUM control charts is considered to be 0.5 .

The following remarks are given in Tables 1-4:

1. The $A R L$ of the combined control charts based on RSS methods is boosted, especially for monitoring small and moderate changes of the mean (Tables $1-4)$;

2. With the assumption that the $A R L_{0}$ is of fixed value, the control limit coefficients $d^{\prime}$ and $m^{\prime}$ are reduced with increase in $\lambda$ (Tables $1-4$ );
3. With the assumption that the $A R L_{0}$ is of fixed value, the UCL coefficient $L$ increases with increase in $\lambda$ (Tables 1-4).

Furthermore, to provide an overview, graphical presentations of ARL are illustrated in Figures 1-6 for comparing the performance of our proposed mixed MEC and MCE control charts under MRSS and DRSS methods with classical CUSUM, EWMA, MEC, and MCE under other existing sampling methods.

As can be seen in Figure 1, it is obvious that where the SRS is used, for large values of $\lambda$ under small shifts, the mixed MEC and MCE control charts outperform both classic EWMA and CUSUM control charts. Given the increased shift for all values of $\lambda$ while the MEC control chart deteriorated performance, the MCE control chart exhibits a relatively acceptable performance.

Figure 2 shows that based on the RSS method, MEC-RSS performs better in detecting small shifts for large values of $\lambda$. Furthermore, for medium and large shifts in all values of $\lambda$, the MCE control chart outperforms other control charts.

Based on Figure 3, first, a comparison of the proposed control charts with the CUSUM-RSS chart illustrates that the proposed MEC-DRSS chart performs much better in detecting small shifts $(\delta \geq 0.5)$ for large values of $\lambda$. By comparing the proposed MCEDRSS control chart with the CUSUM-RSS control chart in terms of performance, it is evident that the new control outperforms the classic CUSUM-RSS control chart in detecting all small, large, and medium changes in all values of $\lambda$. Second, the comparison of the proposed MEC-DRSS control chart with the EWMARSS control chart shows that the proposed control chart performs much better for large values of $\lambda$ in small shifts. Comparison of the performance of the proposed MCE-DRSS control chart with that of the EWMA-RSS control chart illustrates that the proposed control chart enjoys the best performance for all values of $\lambda$ in detecting large shifts $(\delta \geq 0.5)$. Moreover, the comparison of the out-of-control ARL values for the two proposed MEC-DRSS and MCE-DRSS control charts shows that the MEC-DRSS control chart outperforms the MCE-DRSS control chart for following shifts $(\delta \geq 0.25)$. However, to change $\delta \geq 0.5$, the performance of the MCE-DRSS control chart is better.

Figure 4 shows the comparison of the proposed MEC-MRSS control chart. Thus, it can be concluded that the proposed MCE-MRSS control chart for all values of $\lambda$ in detecting all small, large, and medium shifts has better performance than the classic CUSUMRSS in detecting all small, large, and medium shifts. Similarly, the proposed MEC-MRSS control chart performs much better in detecting small shifts $(\delta \geq 0.5)$ for large values of $\lambda$ than the EWMA-RSS control chart. 
Table 1. Out-of-control average run length $\left(A R L_{1}\right)$ characteristics of the new proposed Mixed EWMA-CUSUM (MEC) control chart based on Double Ranked Set Sampling (DRSS) method with in-control average run length $A R L_{0}=200$ and $d^{\prime}=0.5$ under shift from $\mu_{0}$ to $\mu_{0}+\delta \sigma_{0}$.

\begin{tabular}{ccccc}
\hline $\boldsymbol{\delta}$ & $\boldsymbol{\lambda}=\mathbf{0 . 1}$, & $\boldsymbol{\lambda}=\mathbf{0 . 2 5}$, & $\boldsymbol{\lambda}=\mathbf{0 . 5}$, & $\boldsymbol{\lambda}=\mathbf{0 . 7 5}$, \\
& $\boldsymbol{D}^{\prime}=\mathbf{2 3 . 2}$ & $\boldsymbol{D}^{\prime}=\mathbf{1 4 . 3}$ & $\boldsymbol{D}^{\prime}=\mathbf{8 . 5 1}$ & $\boldsymbol{D}^{\prime}=\mathbf{5 . 8 1}$ \\
\hline 0 & 199.62 & 200.66 & 199 & 201.8 \\
0.25 & 15.46 & 11.92 & 10.1 & 9.59 \\
0.5 & 9.45 & 6.57 & 4.85 & 4.13 \\
0.75 & 7.3 & 4.92 & 3.48 & 2.8 \\
1 & 6.14 & 4.08 & 2.86 & 2.19 \\
2 & 4.02 & 2.89 & 1.99 & 1.16 \\
3 & 3.03 & 2 & 1.2 & 1 \\
\hline
\end{tabular}

Table 2. Out-of-control average run length $\left(A R L_{1}\right)$ characteristics of the new proposed Mixed EWMA-CUSUM(MEC) control chart based on Median Ranked Set Sampling (MRSS) method with in-control average run length $A R L_{0}=200$ and $m^{\prime}=0.5$ under shift from $\mu_{0}$ to $\mu_{0}+\delta \sigma_{0}$.

\begin{tabular}{ccccc}
\hline $\boldsymbol{\delta}$ & $\begin{array}{c}\boldsymbol{\lambda}=\mathbf{0 . 1}, \\
\boldsymbol{M}^{\prime}=\mathbf{2 3 . 2}\end{array}$ & $\begin{array}{c}\boldsymbol{\lambda}=\mathbf{0 . 2 5}, \\
\boldsymbol{M}^{\prime}=\mathbf{1 4 . 3}\end{array}$ & $\begin{array}{c}\boldsymbol{\lambda}=\mathbf{0 . 5}, \\
\boldsymbol{M}^{\prime}=\mathbf{8 . 5 6}\end{array}$ & $\begin{array}{c}\boldsymbol{\lambda}=\mathbf{0 . 7 5}, \\
\boldsymbol{M}^{\prime}=\mathbf{5 . 8 3}\end{array}$ \\
\hline 0 & 201.5 & 200.12 & 199.74 & 200.75 \\
0.25 & 17.06 & 13.4 & 11.73 & 11.33 \\
0.5 & 10.27 & 7.21 & 5.46 & 4.7 \\
0.75 & 7.91 & 5.34 & 3.84 & 3.13 \\
1 & 6.62 & 4.38 & 3.1 & 2.4 \\
2 & 4.35 & 2.99 & 2 & 1.44 \\
3 & 3.36 & 2.02 & 1.68 & 1 \\
\hline
\end{tabular}

Table 3. Out-of-control average run length $\left(A R L_{1}\right)$ characteristics of the new proposed Mixed CUSUM-EWMA(MCE) control chart based on Double Ranked Set Sampling (DRSS) method with in-control average run length $A R L_{0}=200$ and $k=0.5$ under the shift from $\mu_{0}$ to $\mu_{0}+\delta \sigma_{0}$.

\begin{tabular}{ccccc}
\hline $\boldsymbol{\delta}$ & $\boldsymbol{\lambda}=\mathbf{0 . 1}$, & $\boldsymbol{\lambda}=\mathbf{0 . 2 5}$, & $\boldsymbol{\lambda}=\mathbf{0 . 5}$, & $\boldsymbol{\lambda}=\mathbf{0 . 7 5}$, \\
& $\boldsymbol{L}=\mathbf{5 . 1 5}$ & $\boldsymbol{L}=\mathbf{5 . 7 6}$ & $\boldsymbol{L}=\mathbf{9 . 8}$ & $\boldsymbol{L}=\mathbf{1 0 . 5 6}$ \\
\hline 0 & 199.48 & 198.6 & 199.68 & 200.41 \\
0.25 & 20.71 & 23.8 & 26.73 & 28.71 \\
0.5 & 3.99 & 4.15 & 4.4 & 4.65 \\
0.75 & 1.8 & 1.8 & 1.8 & 1.84 \\
1 & 1.2 & 1.2 & 1.21 & 1.21 \\
2 & 1 & 1 & 1 & 1 \\
3 & 1 & 1 & 1 & 1 \\
\hline
\end{tabular}

Table 4. Out-of-control average run length $\left(A R L_{1}\right)$ characteristics of the new proposed Mixed CUSUM-EWMA(MCE) control chart based on Median Ranked Set Sampling (MRSS) method with in-control average run length $A R L_{0}=200$ and $k=0.5$ under the shift from $\mu_{0}$ to $\mu_{0}+\delta \sigma_{0}$.

\begin{tabular}{ccccc}
\hline $\boldsymbol{\delta}$ & $\boldsymbol{\lambda}=\mathbf{0 . 1}$, & $\boldsymbol{\lambda}=\mathbf{0 . 2 5}$, & $\boldsymbol{\lambda}=\mathbf{0 . 5}$, & $\boldsymbol{\lambda}=\mathbf{0 . 7 5}$, \\
& $\boldsymbol{L}=\mathbf{4 . 5}$ & $\boldsymbol{L}=\mathbf{6 . 5}$ & $\boldsymbol{L}=\mathbf{8 . 1 5}$ & $\boldsymbol{L}=\mathbf{8 . 8 5}$ \\
\hline 0 & 200.19 & 200.56 & 200.07 & 199.94 \\
0.25 & 20.9 & 24.16 & 27.57 & 29.99 \\
0.5 & 4.54 & 4.72 & 5.02 & 5.29 \\
0.75 & 2.14 & 2.12 & 2.13 & 2.15 \\
1 & 1.42 & 1.40 & 1.38 & 1.38 \\
2 & 1 & 1 & 1 & 1 \\
3 & 1 & 1 & 1 & 1 \\
\hline
\end{tabular}



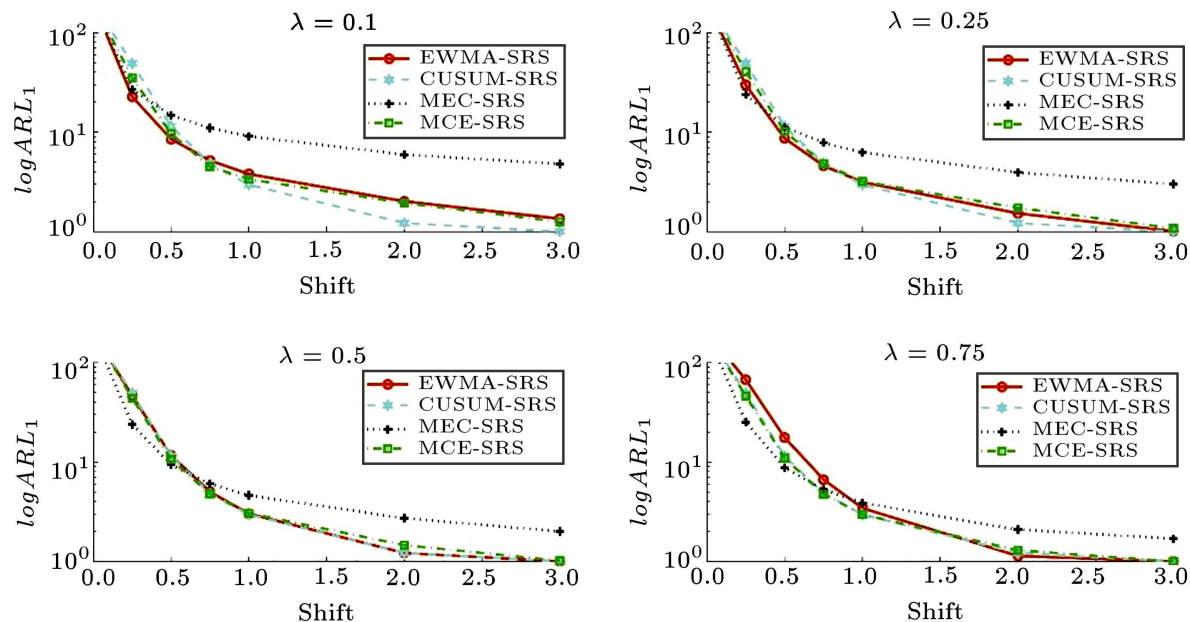

Figure 1. Logarithm of out-of-control average run length $\left(\log A R L_{1}\right)$ for Mixed EWMA-CUSUM (MEC), Mixed CUSUM-EWMA (MCE) and EWMA, CUSUM control charts based on Simple Random Sampling (SRS) method with in-control average run length $A R L_{0}=200$.
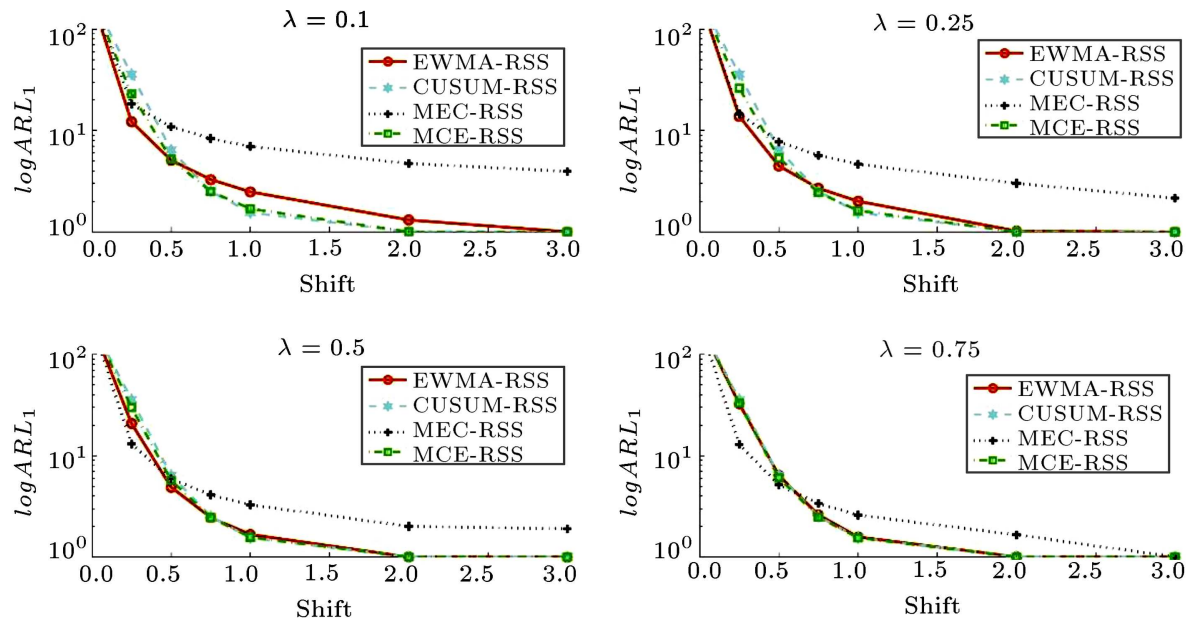

Figure 2. Logarithm of out-of-control average run length $\left(\log A R L_{1}\right)$ for Mixed EWMA-CUSUM (MEC), Mixed CUSUM-EWMA (MCE) and EWMA, CUSUM control charts based on Ranked Set Sampling (RSS) method with in-control average run length $A R L_{0}=200$.
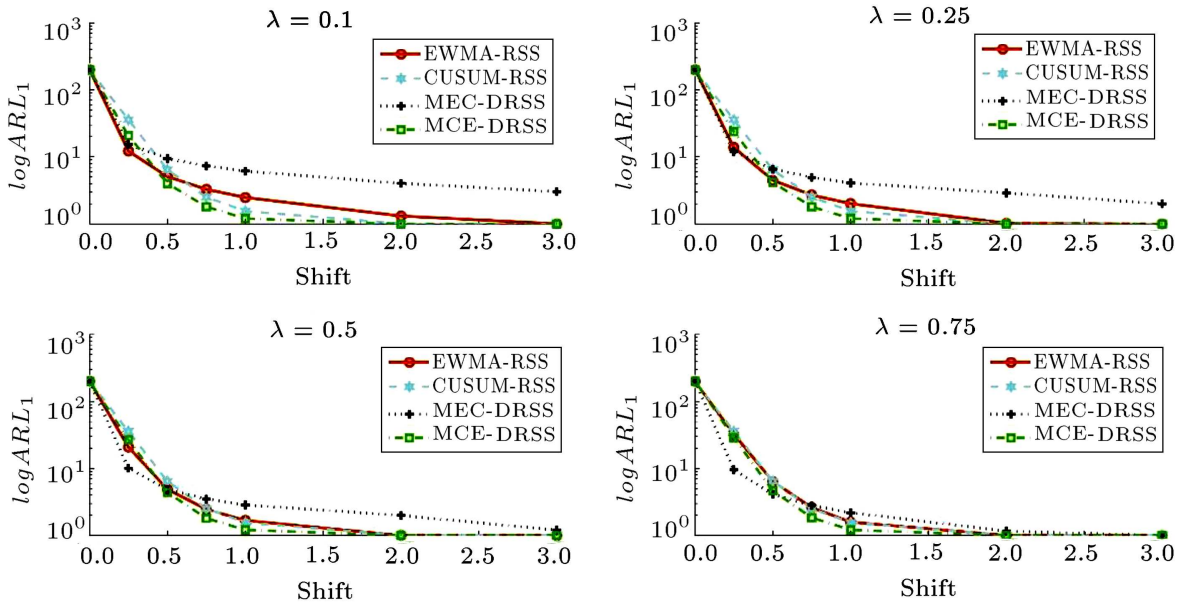

Figure 3. Logarithm of out-of-control average run length $\left(\log A R L_{1}\right)$ for Mixed EWMA-CUSUM (MEC), Mixed CUSUM-EWMA (MCE) control charts based on Double Ranked Set Sampling (DRSS) method and CUSUM, EWMA control charts based on Ranked Set Sampling (RSS) method with in-control average run length $A R L_{0}=200$. 

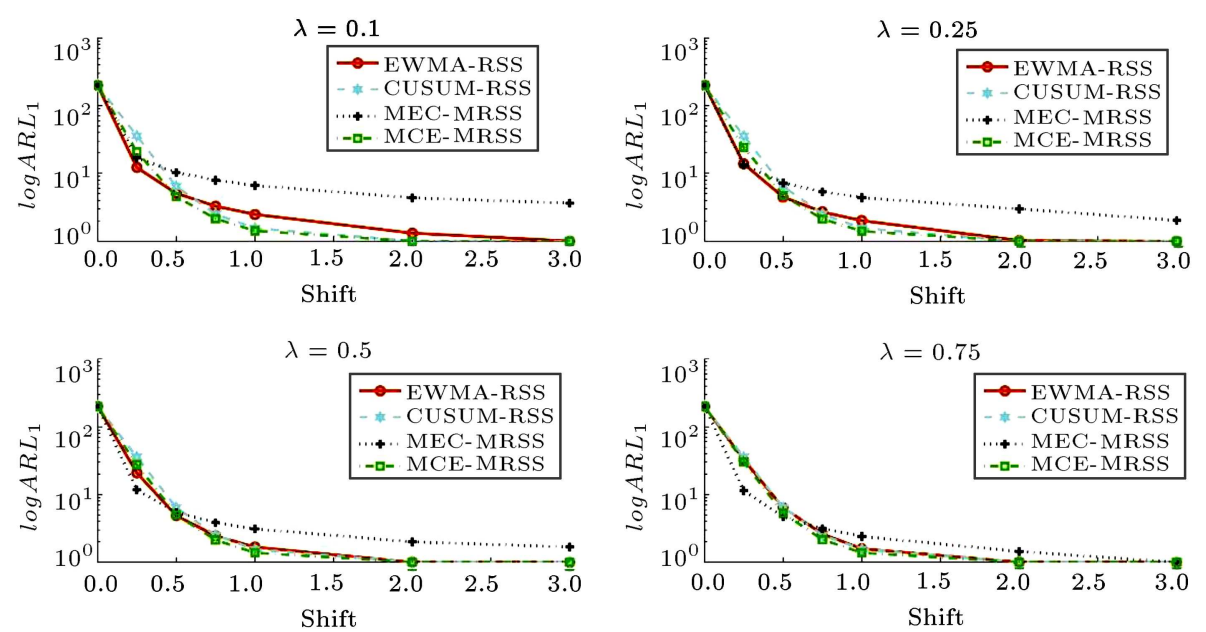

Figure 4. Logarithm of out-of-control average run length $\left(\log A R L_{1}\right)$ for Mixed EWMA-CUSUM (MEC), Mixed CUSUM-EWMA control charts based on Median Ranked Set Sampling (MRSS) method and CUSUM, EWMA control charts based on Ranked Set Sampling (RSS) method with in-control average run length $A R L_{0}=200$.
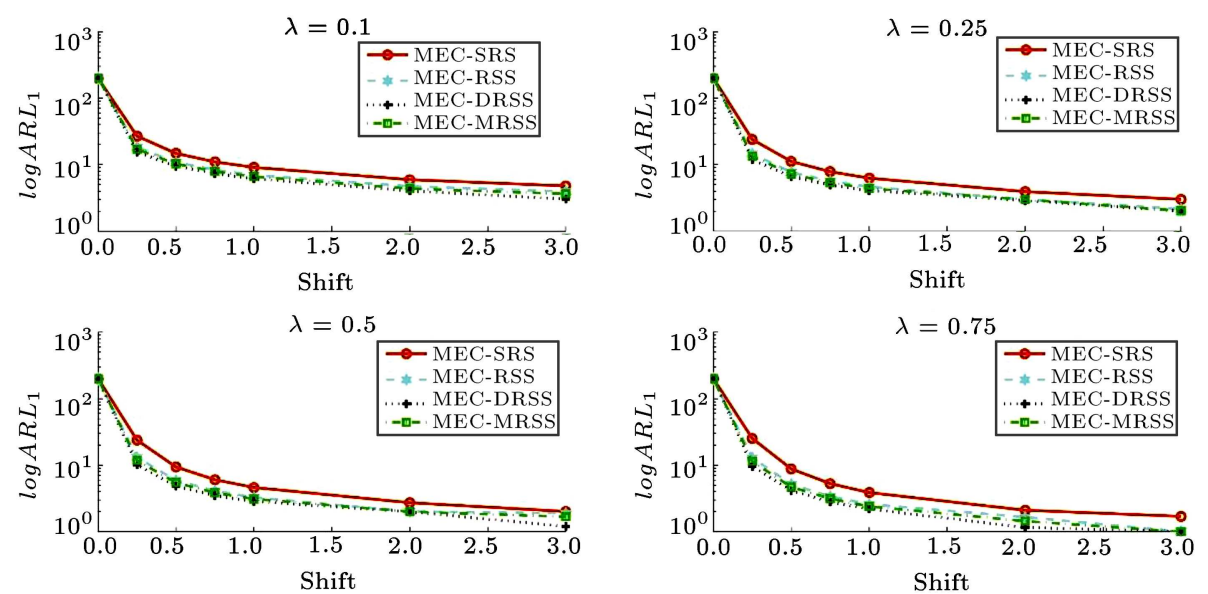

Figure 5. Logarithm of out-of-control average run length $\left(\log A R L_{1}\right)$ for Mixed EWMA-CUSUM (MEC) control charts based on Simple Random Sampling (SRS), Ranked Set Sampling (RSS), Median Ranked Set Sampling (MRSS), Double Ranked Set Sampling (DRSS) methods, with in-control average run length $A R L_{0}=200$.

Also, regarding the proposed MCE-MRSS and EWMARSS control charts, it can be seen that the new control chart performs better at all $\lambda$ values for large shifts $(\delta \geq 0.5)$. Comparison of the out-of-control values for the two proposed MEC-MRSS and MCE-MRSS control charts demonstrates that the MEC-MRSS control chart performs better than the MCE-MRSS control chart for all $\lambda$ values in the $\delta \geq 0.25$ shifts. However, in the case of other shifts $(\delta \geq 0.5)$, the performance of the MCE-MRSS control chart appears to be more efficient.

From Figure 5, it can be seen that the proposed MEC-DRSS control chart at all $\lambda$ values for all shifts has the best performance among MEC-SRS, MECRSS, and MEC-MRSS control charts.

A comparison of the out-of-control values in the proposed control charts and the MEC-RSS control chart is made, as given in Figure 6 . It can be seen that the proposed MCE-DRSS control chart outperforms the MCE-SRS, MCE-RSS, and MCE-MRSS control charts at all $\lambda$ values for detecting all changes.

The overall findings of the proposed chart, as compared to other existing control charts, are summarized as follows:

- The MEC and MCE modified control charts do usually perform better than the classic CUSUM and EWMA control charts in detecting small shifts;

- The proposed MEC-DRSS and MEC-MRSS control charts perform exceptionally well for small shifts; they usually have better performance than EWMA, CUSUM, and MCE control charts. However, the proposed MEC-DRSS and MEC-MRSS control charts are poor in monitoring medium to large mean shifts in the process;

- The proposed MCE-DRSS and MCE-MRSS are better than CUSUM, EWMA, and MEC control 

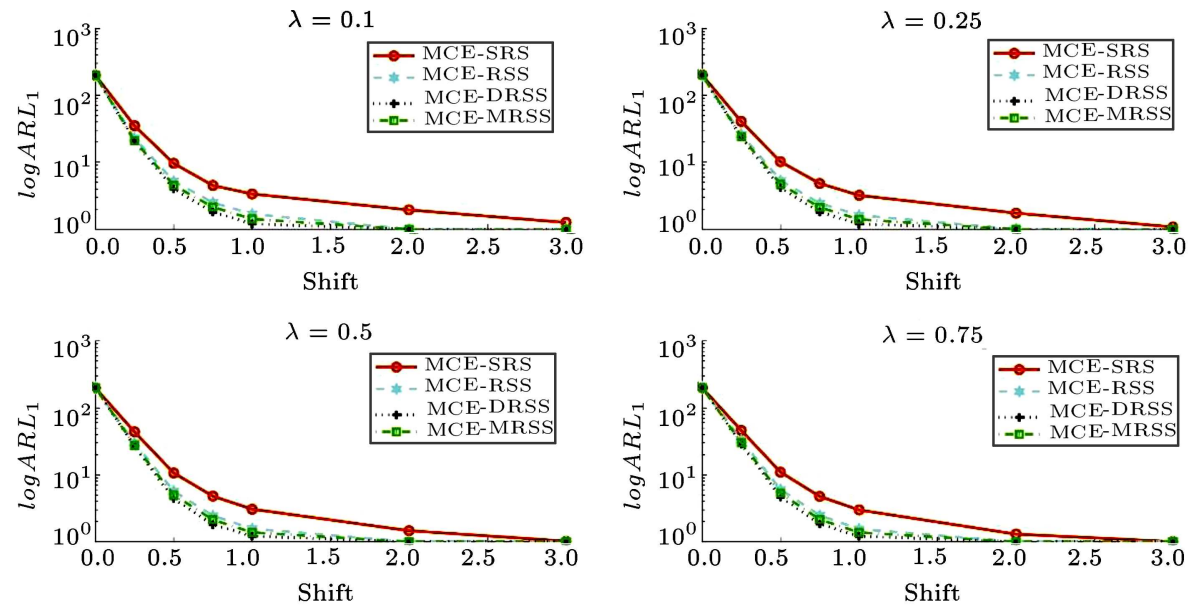

Figure 6. Logarithm of out-of-control average run length $\left(\log A R L_{1}\right)$ for Mixed CUSUM-EWMA (MCE) control charts based on Simple Random Sampling (SRS), Ranked Set Sampling (RSS), Median Ranked Set Sampling (MRSS), Double Ranked Set Sampling (DRSS) methods with in-control average run length $A R L_{0}=200$.
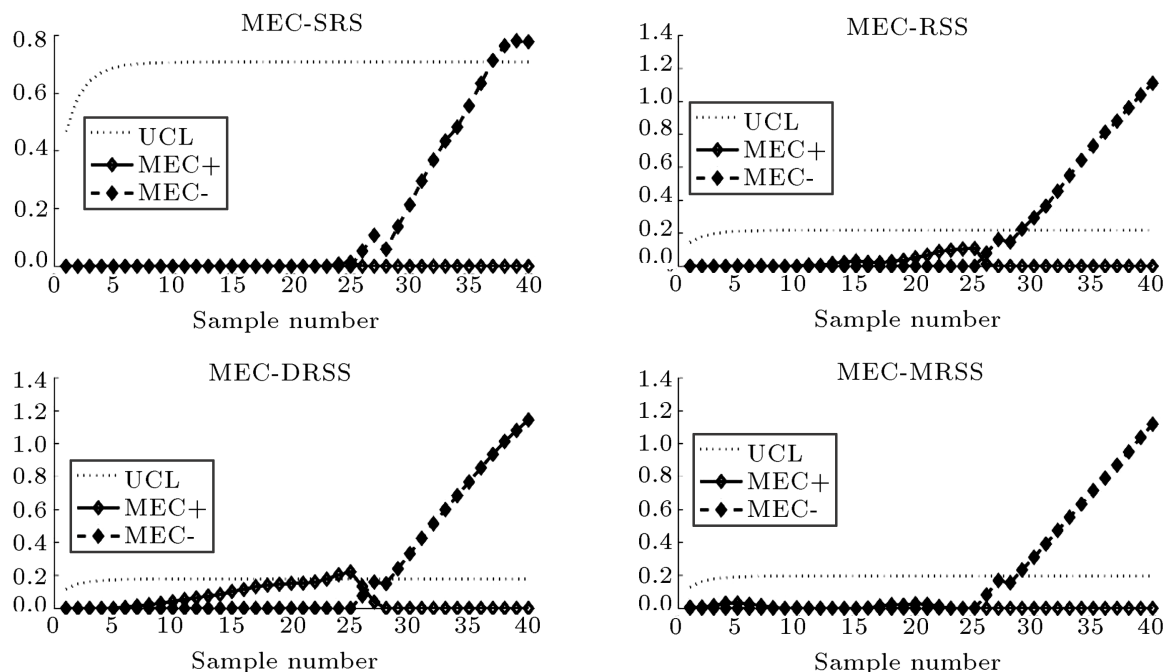

Figure 7. Mixed EWMA-CUSUM (MEC) control charts based on Simple Random Sampling (SRS), Ranked Set Sampling (RSS), Median Ranked Set Sampling (MRSS), Double Ranked Set Sampling (DRSS) methods with in-control average run length $A R L_{0}=200, \lambda=0.25, d^{\prime}=0.5$, and $m^{\prime}=0.5$.

charts in detecting medium and large shifts in the process mean;

- The modified MEC and MCE control charts based on the DRSS scheme outperform the other mixed control charts based on SRS, RSS, and MRSS methods.

\section{A real case}

In this section, to illustrate the application of the proposed control charts under real conditions, we use a real case given in Montgomery [32]. A hard-baking process used in photography to produce semiconductors is selected. During one hour, a data set of 40 observations, each of size 5 , is gathered from the process. Then, all 40 observations are combined to produce 200 data sets.
For each of the SRS, RSS, MRSS, and DRSS methods, 25 samples, each with a size of 5 , are produced under the in-control process parameters and 15 samples under the out-of-control process with a negative shift size of 0.1 using the SRS and the RSS methods (RSS, MRSS, and DRSS). Then, the MEC-SRS, MCE-SRS, MECRSS and MCE-RSS, MEC-DRSS, MCE-DRSS, MECMRSS, and MCE-MRSS control charts are designed to monitor this process. In all of these charts, the value of $A R L_{0}$ is equal to $200(\lambda=0.25)$. Also, the reference value in all CUSUM control charts is considered equal to 0.5 . Figure 7 shows the MEC control charts based on RSS methods, whereas the MCE control charts based on RSS methods are shown in Figure 8.

According to Figure 7, the MEC-SRS and MECRSS control charts show the out-of-control signal from sample 38 and sample 30 , respectively. Upon using the 

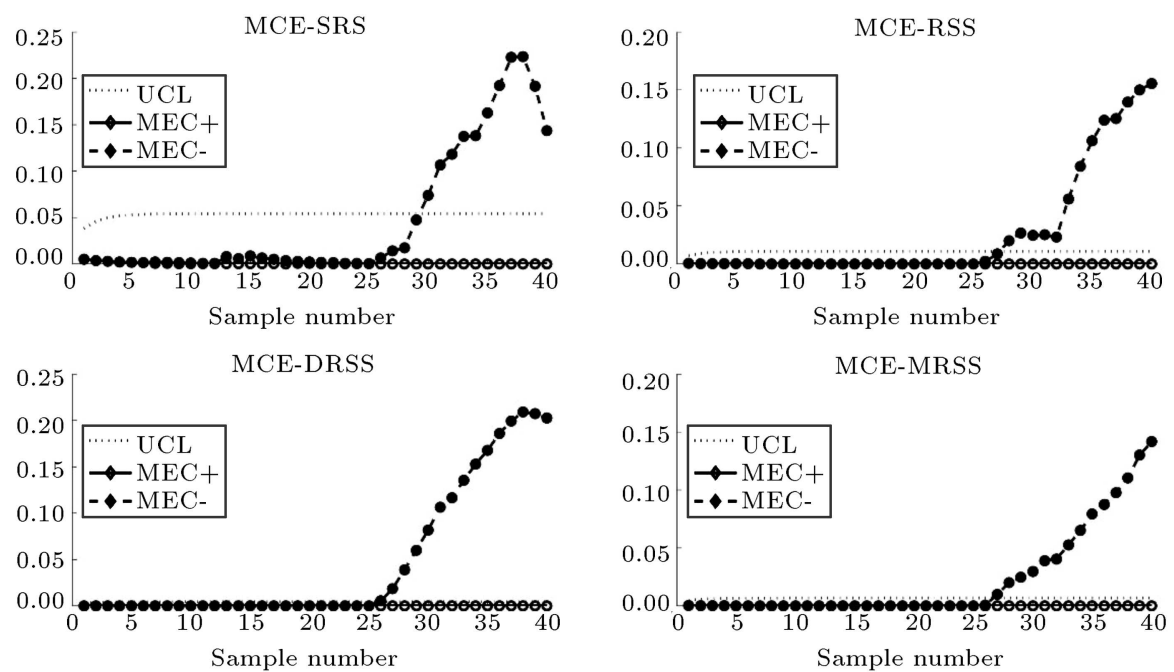

Figure 8. Mixed CUSUM-EWMA (MCE) control charts based on Simple Random Sampling (SRS), Ranked Set Sampling (RSS), Median Ranked Set Sampling (MRSS), Double Ranked Set Sampling (DRSS) methods with in-control average run length $A R L_{0}=200, \lambda=0.25$, and $k=0.5$.

proposed MEC-DRSS and MEC-MRSS control charts, we have received out-of-control signals from samples 27 and 29 , respectively.

Similarly, according to Figure 8, from Sample 30 in the MCE-SRS control chart and Sample 28 in the MCE-RSS control, the out-of-control state is detected. However, the application of the proposed MCE-MRSS and MCE-DRSS control charts shows that out-of-control signals are detected in samples 27 and 26 , respectively.

Based on the analysis of the above-mentioned detection abilities, it is evident that the proposed modified MEC and MCE control charts are of superior performance against existing MEC and MCE control charts based on SRS and RSS methods. Moreover, as both MEC-DRSS and MCE-DRSS detect the outof-control signals more quickly than MEC-MRSS and MCE-MRSS, it can be concluded that the proposed mixed control charts based on DRSS scheme are more effective than the control charts based on MRSS in detecting changes in the mean process.

\section{Conclusion and suggestions for future research}

This study employed Double Ranked Set Sampling (DRSS) and Median Ranked Set Sampling (MRSS) methods to design Mixed EWMA-CUSUM (MEC) and Mixed CUSUM-EWMA (MCE) control charts for monitoring the mean of a normal process. The performances of new control charts and similar control charts were comparatively evaluated using Ranked Set Sampling (RSS) and Simple Random Sampling (SRS) methods in a numerical example by simulation. Also, the performance of the proposed control charts was compared with each of the classic control charts. The results indicated that the MEC and MCE control charts performed well in detecting small to medium process shifts. Besides, the use of a DRSS model in both mixed control charts improved the performance of the proposed control charts. For future studies, one can develop mixed control charts using other types of sampling methods for monitoring both mean and variability. Also, nonparametric control charts can be designed with different sampling methods under nonnormal data.

\section{References}

1. Shewhart, W.A. "Some applications of statistical methods to the analysis of physical and engineering data", Bell System Technical Journal, 3(1), pp. 43-87 (1924).

2. Roberts, S.W. "Control chart tests based on geometric moving averages", Technometrics, 1(3), pp. 239-250 (1959).

3. Page, E.S. "Cumulative sum charts", Technometrics, 3(1), pp. 1-9 (1951).

4. Lucas, J.M. "Combined shewhart-cusum quality control schemes", Journal of Quality Technology, 14(2), pp. 51-59 (1982).

5. Lucas, J.M. and Saccucci, M.S. "Exponentially weighted moving average control schemes properties and enhancements", Technometrics, 32(1), pp. 1-12 (1990).

6. Jiang, W., Shu, L., and Apley, D.W. "Adaptive CUSUM procedures with EWMA-based shift estimators", IIE Transactions, 40(10), pp. 992-1003 (2008).

7. Abbas, N., Riaz, M., and J.M.M, R. "Mixed exponentially weighted moving average-cumulative sum charts for process monitoring", Quality and Reliability Engineering International, 29(3), pp. 345-356 (2013). 
8. Riaz, M., Zaman, B., Abbas, N., et al. "Mixed cumulative sum-exponentially weighted moving average control charts: an efficient way of monitoring process location", Quality and Reliability Engineering International, 31(8), pp. 1407-1421 (2015).

9. Aslam, M.A. "Mixed EWMA-CUSUM control chart for weibull-distributed quality characteristics", Quality and Reliability Engineering International, 32(8), pp. 2987-2994 (2016).

10. Osei-Aning, R., Abbasi, S.A., and Riaz, M. "Mixed EWMA-CUSUM and mixed CUSUM-EWMA modified control charts for monitoring first order autoregressive processes", Quality Technology \& Quantitative Management, 14(4), pp. 429-453 (2017).

11. Riaz, M., Khaliq, Q.U.A., and Gul, S. "Mixed Tukey EWMA-CUSUM control chart and its applications", Quality Technology \& Quantitative Management, 14(4), pp. 429-453 (2017).

12. Ajadi, J.O. and Riaz, M. "Mixed multivariate EWMACUSUM control charts for an improved process monitoring", Communications in Statistics - Theory and Method, 46(14), pp. 6980-6993 (2017).

13. Aslam, M., Azam, M., and Jun, C.H. "A HEWMACUSUM control chart for the Weibull distribution", Communications in Statistics - Theory and Methods, 47(24), pp. 5973-5985 (2018).

14. Abbas, N., Raji, I.A., Riaz, M., et al. "Designing mixed EWMA Dual-CUSUM chart with applications in petro-chemical industry", IEEE Access, 6, pp. 78931-78946 (2018).

15. Riaz, M., Ajadi, J.O., Mahmood, T., et al. "Multivariate mixed EWMA-CUSUM control chart for monitoring the process variance-covariance matrix", IEEE Access, 7, pp. 100174-100186 (2019).

16. Rao, G.S., Aslam, M., and Rasheed, U. "Mixed EWMA-CUSUM chart for COM-Poisson distribution", Journal of Statistics and Management Systems, 23(3), pp. 511-527 (2020).

17. McIntyre, G.A. "A method for unbiased selective F, using ranked sets", Australian Journal of Agriculture Research, 3(4), pp. 385-390 (1952).

18. Salazar, R.D. and Sinha, A.K. "Control chart x-bar based on ranked set sampling", Comunicacion Tecica,, No. 1-97-09 (PE/CIMAT) (1977).

19. Muttlak, H.A. and Al-Sabah, W.S. "Statistical quality control based on ranked set sampling", Journal of Applied Statistics, 30(9), pp. 1055-1078 (2003).

20. Abujiya, M.R. and Muttlak, H.A. "Monitoring the process mean and variance using ranked set sampling and its modifications", Journal of Statistical Theory and Applications, 6(4), pp. 408-422 (2007).
21. Mehmood, R., Riaz, M., and Does, R.J.M.M. "Control charts for location based on different sampling schemes", Journal of Applied Statistics, 40(3), pp. 483-494 (2013).

22. Al-Sabah, W.S. "Cumulative sum statistical control charts using ranked set sampling data", Pakistan Journal of Statistics, 26(2), pp. 365-378 (2010).

23. Abujiya, M.R. and Lee, M.H. "The three statistical control charts using ranked set sampling", 5th International Conference on Modeling, Simulation and Applied Optimization (ICMSAO) (2013). DOI: 10.1109/ICMSAO.2013.6552684

24. Haq, A., Brown, J., and Moltchanova, E. "Improved exponentially weighted moving average control charts for monitoring process mean and dispersion", Quality and Reliability Engineering International, 31(2), pp. 217-237 (2015).

25. Awais, M. and Haq, A. "An EWMA chart for monitoring process mean", Journal of Statistical Computation and Simulation, 88(5), pp. 1003-1025 (2018).

26. Haq, A. and Munir, W. "Improved CUSUM charts for monitoring process mean", Journal of Statistical Computation and Simulation, 88(9), pp. 1684-1701 (2018).

27. Abbasi, S.A. "Location charts based on ranked set sampling for normal and non-normal processes", Published online in Quality and Reliability Engineering International (2019). DOI: 10.1002/qre.2463

28. Cochran, W.G., Sampling Techniques, 3rd Edition, John Wiley \& Sons (1997).

29. Wolfe, D.A. "Ranked set sampling: An approach to more efficient data collection", Statistical Science, 19(4), pp. 636-643 (2004).

30. Al-Saleh, M.F. and Al-Kadiri, M.A. "Double-ranked set sampling", Statistics \& Probability Letters, 48(2), pp. 205-212 (2000).

31. Muttlak, H.A. "Median ranked set sampling", Journal of Applied Statistical Sciences, 66, pp. 245-255 (1997).

32. Montgomery, D.C., Introduction to Statistical Quality Control, 6th Edition, John Wiley \& Sons, New York (2009).

\section{Biographies}

Atieh Mohammadkhani holds an MS degree in Industrial Engineering from Shahed University in Iran. Her research interests are statistical process monitoring and quality control.

Amirhossein Amiri is an Full Professor at Shahed University in Iran. He holds BS, MS, and PhD in Industrial Engineering from Khajeh Nasir University of Technology, Iran University of Science and Technology, and Tarbiat Modares University in Iran, respectively. He is now the Director of postgraduate education at Shahed University. His research interests are statistical 
quality control, profile monitoring, and six sigma. He has published many papers in the area of statistical process monitoring in high-quality international journals such as Quality and Reliability Engineering International, Communications in Statistics, Comput- ers and Industrial Engineering, Journal of Statistical Computation and Simulation, Soft Computing, and so on. He has also published a book with John Wiley and Sons in 2011 entitled Statistical Analysis of Profile Monitoring. 